\title{
Análisis de agua no contabilizada en el sistema de abastecimiento urbano del municipio de Facatativá, Colombia
}

\author{
Analysis of non-revenue water in the urban supply system of the \\ municipality of Facatativá, Colombia
}

\author{
Diego Bueno-Herrera $^{1}{ }^{\mathbb{D}}$, Edison Monroy-Ávila² ${ }^{\mathbb{D}}$, Carlos Zafra-Mejía ${ }^{3}$ iD
}

Cómo citar: Bueno-Herrera, D., Monroy-Ávila, E. y Zafra-Mejía, C. (2020). Análisis de agua no contabilizada en el sistema de abastecimiento urbano del municipio de Facatativá, Colombia. Tecnura, 24(63) 73-87. DOI: https:// doi.org/10.14483/22487638.15333

Fecha de de recepción: 19 de junio de 2019

\section{RESUMEN}

Contexto: Las pérdidas de agua son un obstáculo financiero para empresas prestadoras del servicio de abastecimiento, ya que generan pérdidas económicas que son trasladadas a los usuarios, elevando de esta manera los costos de suministro del agua. El objetivo de este artículo es presentar un análisis del agua no contabilizada en un sistema de abastecimiento urbano en Colombia.

Método: Este estudio se realizó a partir de información del agua producida (AP) y facturada por el sistema de abastecimiento durante cerca de 16 años. Se realizó un análisis comparativo con respecto a reportes de agua no contabilizada en sistemas colombianos e internacionales de referencia. Se evaluó también la influencia del régimen de precipitación sobre el índice de
Fecha de de aceptación: 18 de octubre de 2019

agua no contabilizada (IANC). Se realizó finalmente un análisis económico prospectivo mediante modelos ARIMA.

Resultados: Se observa un incremento del 5,0 $\%$ en el IANC por cada 100000 m3 de AP. Existe un incremento del 2,28\% en el IANC por cada incremento de $100 \mathrm{~mm}$ en la precipitación anual. El sistema posee un menor precio en el AP $(22,2 \%)$ con respecto a sistemas latinoamericanos de referencia. Sin embargo, el sistema posee un mayor precio (33,3 \%) en relación con el promedio colombiano. El análisis ARIMA muestra que los efectos del AP en un instante de tiempo determinado se transfieren durante seis meses en el sistema de abastecimiento.

Conclusiones: La aplicación del IANC en sistemas de abastecimiento es una herramienta útil

1 Ingeniero civil, especialista en Agua y Saneamiento Ambiental. Escuela de Posgrados, Universidad Manuela Beltrán. Bogotá, Colombia. Contacto: diegob693@gmail.com

ORCID: https://orcid.org/0000-0002-2086-3781

2 Ingeniero ambiental, especialista en Agua y Saneamiento Ambiental, magíster en Tecnologías para el Manejo de Aguas y Residuos. Escuela de Posgrados, Universidad Manuela Beltrán. Bogotá, Colombia. Contacto: ingenierofabianm@gmail.com ORCID: https://orcid.org/0000-0003-3757-9442

3 Ingeniero civil, magíster en Ingeniería Ambiental, doctor en Ingeniería Ambiental. Grupo de Investigación en Ingeniería Ambiental (GllAUD), Universidad Distrital Francisco José de Caldas. Bogotá, Colombia. Contacto: czafra@udistrital.edu.co

ORCID: https://orcid.org/0000-0002-4061-4897 
para el estudio y desarrollo de programas de control de pérdidas de agua.

Palabras clave: acueducto; agua no contabilizada; ARIMA; sistema de abastecimiento.

\section{ABSTRACT}

Context: Water losses are a financial obstacle for supply service providers as they generate economic losses that are passed on to users, thereby raising water supply costs. The objective of this paper is to shows an analysis of non-revenue water in an urban supply system in Colombia.

Method: This study was conducted from information of water produced (WP) and billed by the supply system for about 16 years. A comparative analysis was carried out with respect to reports of non-revenue water in Colombian and international reference systems. Rainfall influence on the non-revenue water index (NRWI) was also assessed. A prospective economic analysis was finally carried out using ARIMA models.

Results: There is an increase of $5.0 \%$ in NRWI per $100000 \mathrm{~m} 3$ of WP. There is an increase of $2.28 \%$ in NRWI for each increase of $100 \mathrm{~mm}$ in annual rainfall. The system has a lower price on WP $(22.2 \%)$ in relation with Latin American reference systems. However, the system has a higher price $(33.3 \%)$ relative to the Colombian average. Analysis ARIMA shows that the effects of AP in an instant of time are transferred during six months in the water supply system.

Conclusions: The application of NRWI in supply systems is a useful tool for the study and development of water loss control programs.

Keywords: Aqueduct; Non-revenue water; ARIMA; Water supply system.

\section{INTRODUCCIÓN}

Las pérdidas de agua en sistemas de abastecimiento posiblemente tuvieron un impacto ambiental negativo por la sobreexplotación del recurso hídrico y, en lo financiero, conIlevaron probablemente al incremento de tarifas por prestación del servicio debido a las elevadas inversiones económicas para cubrir estos costos operacionales y administrativos (Tabesh et al., 2018). Las investigaciones a nivel mundial indicaron que, de seguir las prácticas actuales de consumo de agua, enfrentaremos una escasez mundial del $40 \%$, entre la demanda prevista y el suministro disponible para el año 2030 (Agatón et al., 2016; Salimi et al., 2018). Por ejemplo, se estimó para el año 2013 que 780 millones de habitantes no tuvieron acceso a agua potable segura, y que en algunos países esto provocó la muerte de grupos sensibles (niños y ancianos) y pérdidas anuales de hasta el 7,0 \% del PIB (The World Bank, 2013; Pérez, Flores,
González y Mota, 2019). En el mundo, más de 32 billones de $\mathrm{m}^{3}$ de agua potable se fugaron de sistemas de abastecimiento al año, con un costo asociado superior a los USD 18. 000 millones (OSE, 2019).

En el contexto latinoamericano, el Banco Mundial (2013) estimó en promedio que el $45 \%$ del agua producida correspondió a agua no facturada. El caso colombiano no fue la excepción, Ojeda y Arias (2000), y García y Benavides (2019) reportaron en promedio que las pérdidas de agua fueron posiblemente superiores al $33 \%$ y $43 \%$ en los sistemas de abastecimiento municipales, respectivamente. García, Vargas y Granados (2013) también reportaron la escasez de estudios publicados que reunieran y analizaran los datos de pérdidas de agua en empresas prestadoras del servicio de acueducto. Esta carencia de información pudo afectar el proceso de control, regulación y las propuestas de gestión que se plantearon para la disminución de las pérdidas del líquido en los sistemas de abastecimiento latinoamericanos. 
A partir de lo anterior, es necesario entonces desarrollar investigaciones que estudien los factores que incrementan las pérdidas de agua en sistemas de abastecimiento para poder tener acueductos más eficientes y sustentables. En este sentido, el porcentaje de pérdidas en función del índice de agua no contabilizada (IANC) es tal vez uno de los métodos de mayor utilidad para evaluar financiera y técnicamente estos sistemas de abastecimiento. Este índice permitirá desarrollar programas más eficientes para la prevención, reducción y control de pérdidas, disminuyendo así los costos de distribución del agua.

El IANC en las empresas de acueducto es un indicador que representa el porcentaje de pérdidas de agua y refleja la eficiencia con la que opera el sistema (González-Gómez, García-Rubio y Guardiola, 2011). Se calcula como la diferencia porcentual entre el caudal que ingresa y el caudal que sale de una etapa productiva (Tabesh, Yekta y Burrows, 2009). Las pérdidas de agua en un acueducto se clasifican en dos tipos: comerciales y técnicas. Asimismo, las primeras se pueden dividir en: conexiones ilegales, facturación de volúmenes menores a los reales cuando los medidores no funcionan adecuadamente o son alterados, y ausencia de medidores. Por otro lado, las pérdidas técnicas son aquellas que se generan por fugas en accesorios, tuberías fisuradas, rebosaderos y tanques de almacenamiento (Kanakoudis y Gonelas, 2016; Cherchi et al., 2015). Las pérdidas de agua se pueden reducir, pero difícilmente se lograría llegar a eliminarlas en su totalidad. No obstante, es necesario proponer programas de gestión para la detección de fugas, usuarios fraudulentos y fallas en el sistema de acueducto (Boztaş, Özdemir, Durmuşçelebi y Firat, 2019).

En el ámbito internacional existe evidencia del interés por reducir las pérdidas de agua en sistemas de abastecimiento. Por ejemplo,
Dogo et al. (2019) y Venkatesh (2012) reportaron que Dinamarca ha sido capaz de reducir sus pérdidas en $9,0 \%$ mediante un proceso que inició en 1989, con un impuesto sobre el agua producida, el cual les brindó a los proveedores incentivos para reducir las pérdidas y mejorar los sistemas de abastecimiento. Ho, Lin y Lo (2010) desarrollaron una metodología técnica mediante la integración de un modelo de red neuronal artificial basado en sísmica y sistemas de información geográfica, para estudiar las fugas de agua en un sistema de abastecimiento. Nazif, Karamouz, Tabesh y Moridi (2010) también implementaron un modelo de optimización para correlacionar el nivel del agua en los tanques de almacenamiento con las pérdidas de agua en sistemas de distribución.

Una de las metodologías más reconocidas para evaluar las pérdidas de agua en sistemas de abastecimiento (técnicas y comerciales) de países en vías de desarrollo fue la propuesta por la Asociación Internacional del Agua (IWA, por su sigla en inglés). Esta metodología se fundamentó en el desarrollo de un balance de masas del sistema, el cual permitió conocer las condiciones técnicas de funcionamiento, determinar los índices de pérdidas (IANC) en los subsistemas que lo constituyeron, y formular un programa de disminución y control de pérdidas de agua (Liemberger y Wyatt, 2018). En este sentido, la Comisión de Regulación de Agua Potable de Colombia adoptó el IANC (Resolución CRA 315 de 2009) como el indicador de pérdidas para los sistemas de abastecimiento (Castillo, 2014).

El objetivo principal de este artículo es presentar un análisis del agua no contabilizada mediante el IANC en un sistema de abastecimiento urbano en Colombia. Este estudio se realiza a partir de información recolectada durante 15,7 años (2000-2015). Se presenta también un análisis comparativo con respecto a los reportes 
de agua no contabilizada de otras localidades nacionales e internacionales de referencia. Se evalúa además la influencia de las condiciones climáticas sobre el IANC. Finalmente, se expone un análisis económico prospectivo mediante un estudio estadístico de series de tiempo.

\section{MATERIALES Y MÉTODOS}

\section{Descripción del lugar}

El lugar de investigación se localizó en el municipio de Facatativá, en el extremo occidental de la sabana de Bogotá, Colombia $\left(4^{\circ} 48^{\prime} 46^{\prime \prime} \mathrm{N}\right.$, $74^{\circ} 21^{\prime} 00^{\prime \prime}$ O). La zona se caracterizó por una elevación promedio de $2586 \mathrm{~m} \mathrm{~s}$. n. m. y una temperatura media anual de $13,4{ }^{\circ} \mathrm{C}$, con una variación entre $0-22{ }^{\circ} \mathrm{C}$ en el mes más seco. La población estimada para el año 2015 fue de 112600 habitantes. La cobertura del servicio de acueducto fue del $100 \%$ en el casco urbano, y $10 \%$ en el sector rural del municipio. El sistema contabilizó 26576 usuarios para el año 2015, suministrando un caudal promedio de $472530 \mathrm{~m}^{3} / \mathrm{mes}$. Los usuarios facturados se clasificaron de la siguiente manera: residenciales $=24$ 470, comerciales $=1839$, oficiales $=$ 236 , y especiales $=30$. El sistema de acueducto contó con dos plantas de potabilización (El Gatillo y La Guapucha). Estas fueron de tipo convencional con un caudal de diseño de 280 y $30 \mathrm{l} / \mathrm{s}$, respectivamente. La red de distribución de agua antigua del municipio fue construida con tubería de asbesto-cemento, hierro fundido y hierro galvanizado. Algunas de estas tuberías fueron remplazadas por tubería de PVC.

\section{Recolección de información}

La información utilizada para este estudio correspondió a los registros de caudal de agua producida (AP) en la planta de potabilización y caudal de agua facturada (AF) por los usuarios entre el 01/01/2000-31/08/2015. En la recolección de información se utilizaron como referentes metodológicos los tra- bajos realizados por Kanakoudis y Gonelas (2016), y Liemberger y Wyatt (2018). Los registros de AP tuvieron una frecuencia de recolección diaria y los de AF, cada dos meses. De esta manera, la escala temporal de este estudio para el análisis de agua no contabilizada en el sistema fue bimestral. Todos los registros empleados fueron suministrados por la empresa prestadora del servicio de abastecimiento de agua de la municipalidad en estudio. La información climática (precipitación mensual) se tomó del Instituto de Hidrología, Meteorología y Estudios Ambientales de Colombia (Ideam), y correspondió a las siguientes estaciones climatológicas localizadas en el interior del municipio de Facatativá: Tisquesusa $\left(4^{\circ} 48^{\prime} 20,5^{\prime \prime} \mathrm{N} ; 74^{\circ} 17^{\prime} 3^{\prime \prime} \mathrm{W}\right)$, Tesoro $\left(4^{\circ} 48^{\prime} 0,12^{\prime \prime} \mathrm{N} ; 74^{\circ} 19^{\prime} 3,9^{\prime \prime} \mathrm{W}\right)$ y Venecia (451'1,2" N; 74² $\left.4^{\prime} 26,9^{\prime \prime} \mathrm{W}\right)$.

El costo promedio de referencia para el análisis económico prospectivo en la presente investigación fue de USD 0,38 por $\mathrm{m}^{3}$ de AP, el cual correspondió a septiembre de 2015. Este costo promedio de AP fue suministrado por la empresa prestadora del servicio de abastecimiento. La recolección de información para el análisis económico prospectivo se realizó teniendo como referente metodológico el trabajo realizado por Cherchi et al. (2015). La información de costos de AP para otras localidades colombianas e internacionales de referencia fueron tomadas del estudio colombiano sectorial de servicios públicos domiciliarios de acueducto y alcantarillado (Robayo et al., 2017) y del informe del Banco Mundial sobre tarifas de agua potable y alcantarillado en América Latina (The World Bank, 2005).

\section{Análisis de información}

La variación temporal de agua no contabilizada en el sistema de abastecimiento fue estudiada entre 01/01/2000-31/08/2015. Al respecto, se identificaron los periodos de tiempo de máxima y mínima agua no contabilizada. En este 
análisis se utilizó el índice IANC (ecuación (1)) (González-Gómez, García-Rubio y Guardiola, 2011; Tabesh, Yekta y Burrows, 2009):$$
\operatorname{IANC}(\%)=(\mathrm{AP}-\mathrm{AF}) / \mathrm{AP} * 100
$$

Donde, IANC representó el porcentaje de agua no contabilizada en el sistema de abastecimiento; AP, el volumen de agua producido en la potabilizadora a nivel bimestral $\left(\mathrm{m}^{3}\right)$, y $\mathrm{AF}$, el volumen de agua facturado por los usuarios a nivel bimestral $\left(\mathrm{m}^{3}\right)$. Aquí se utilizó también el índice de agua no contabilizada per cápita $(I A N C P=$ IANC por cada 100000 habitantes) y el índice de pérdidas por usuario facturado (IPUF $=\mathrm{m}^{3} /$ suscriptor*mes) (Castillo, 2014; Tabesh, Yekta y Burrows, 2009).

Adicionalmente, se estudió la posible relación entre el consumo de agua (AF) y el número de usuarios registrados, con el objeto de evaluar la variación temporal de la dotación en el sistema de abastecimiento (litros/ habitante*día). Esta dotación fue comparada con la reportada por referentes bibliográficos colombianos: Reglamento técnico para el sector de agua potable y saneamiento básico - RAS (MDE, 2000) y la Resolución 2320 de 2009 por la cual se modificó parcialmente el RAS (MAVDT, 2009). Asimismo, se evaluó la posible relación entre la dotación y el IANC. Se hizo también un análisis comparativo del IANC entre el sistema de abastecimiento en estudio y nueve municipios colombianos de referencia con población urbana entre 8000 y 540000 habitantes: Gachancipá, Pitalito, Mosquera, Sogamoso, Zipaquirá, Tunja, ViIlavicencio, Soacha e Ibagué.

En este estudio, se analizó la influencia del régimen de precipitación sobre el índice IANC. Se recurrió a los registros mensuales de precipitación de las tres estaciones climatológicas seleccionadas (Tisquesusa, Tesoro y Venecia). El régimen de precipitación mensual $(\mathrm{P})$ fue estudiado mediante los periodos propuestos por el método de quintiles $\left(\mathrm{Q}_{\mathrm{i}}\right)$ de Bolognesi (Bohn, Piccolo y Perillo, 2011). Este método consideró los siguientes periodos mensuales de precipitación $(\mathrm{P})$ : muy seco $\left(\mathrm{P}<\mathrm{Q}^{1}\right)$, seco $\left(\mathrm{Q}^{1} \leq \mathrm{P}<\mathrm{Q}^{2}\right)$, normal $\left(\mathrm{Q}^{2} \leq \mathrm{P}<\mathrm{Q}^{3}\right)$, lluvioso $\left(\mathrm{Q}^{3} \leq \mathrm{P}<\mathrm{Q}^{4}\right)$, y muy lluvioso $\left(\mathrm{Q}^{3} \leq \mathrm{P}<\mathrm{Q}^{5}\right)$. Se estudiaron también los periodos anuales de incremento y disminución de la precipitación asociados con el fenómeno climático de Oscilación del Sur: El Niño y La Niña (Díaz, Escobar y Serna, 2014).

Finalmente, se llevó a cabo un análisis económico que incluyó una comparación de los costos de AP (USD por $\mathrm{m}^{3}$ ) en relación con sistemas de abastecimiento de seis países latinoamericanos de referencia. Para esto se tuvo como referente el estudio latinoamericano del Banco Mundial en esta materia (The World Bank, 2005). Se determinó también el costo de agua no facturada durante el periodo de estudio.

Posteriormente, se adelantó un análisis económico prospectivo mediante modelos autorregresivos, integrados y de promedios móviles (ARIMA, por su acrónimo en inglés). Este análisis se desarrolló a corto (un año), mediano (cinco años) y largo plazo (20 años), periodo durante el cual se tuvieron en cuenta como referentes metodológicos las investigaciones de Venkatesh (2012) y Cherchi et al. (2015). La tasa de cambio promedio de pesos colombianos (COP) a dólar americano (USD) para la época de estudio fue de COP 3068,34 por cada dólar.

\section{Análisis estadístico}

La distribución normal de las series de tiempo fue evaluada con una prueba de Kolmogorov-Smirnov ( $p$-valor $>0,05)$. Se utilizaron el coeficiente de correlación de Pearson y la 
prueba t-Student para estudiar las posibles relaciones y diferencias entre variables, respectivamente. Se adoptaron también modelos ARIMA con la metodología de Box-Jenkins (1990) para estudiar las series de tiempo bimestrales de AP y AF. Para los modelos ARIMA se tuvieron en cuenta las tres etapas reportadas por Guerrero (2003): identificación, estimación y verificación del modelo. Durante la etapa de identificación del modelo se determinaron los órdenes de los polinomios autorregresivos $(p)$ y de promedios móviles $(q)$, así como también el grado de diferenciación (q) para cancelar la no estacionalidad de las series de tiempo en estudio. Es decir, se determinaron los órdenes $p, d y q$ del modelo ARIMA. En la segunda etapa se determinaron los parámetros de los polinomios autorregresivos $\left(\varnothing_{-} 1, \ldots, \varnothing_{-} p\right)$ y de promedios móviles $\left(\theta \_1, \ldots, \theta \_\right.$p) para cada modelo identificado por el método de máxima verosimilitud. Finalmente, se verificaron los ocho supuestos propuestos por Box-Jenkins (1990) con el fin de seleccionar los mejores modelos ARIMA para cada serie de tiempo en estudio. Todas las anteriores etapas se trabajaron en el programa informático IBM-SPSS V. 18.0.

\section{RESULTADOS Y DISCUSIÓN}

\section{Agua no contabilizada}

En promedio mensual, los resultados mostraron que durante todo el periodo de investigación el AP por el sistema de abastecimiento fue de $472530 \mathrm{~m}^{3}$; mientras que el AF fue de $308850 \mathrm{~m}^{3}$ (figura 1). Es decir, el sistema dejó de facturar en promedio por mes 163 $680 \mathrm{~m}^{3}$, lo cual representó un porcentaje de agua no contabilizada del 34,6\% (IANC). La prueba t-Student permitió evidenciar diferencias significativas entre el AP y AF ( $p$-valor < $0,001)$. En relación con la variación temporal del IANC, los hallazgos mostraron que entre los meses de marzo y abril de 2010 se re- gistró el mayor IANC (44,7 \%) durante todo el periodo de estudio. Durante estos meses también se observó la mayor AP (542647 m³) y la menor AF en el sistema (299 $945 \mathrm{~m}^{3}$ ). De esta manera, los resultados sugirieron que cuando ocurrió la mayor producción de agua también ocurrió la menor facturación. Es decir, a medida que se incrementó la disponibilidad de agua en el sistema probablemente también se incrementó el porcentaje de agua no contabilizada.

A partir de lo anterior, se realizó un análisis de correlación lineal de Pearson entre el IANC $(\%)$ y AP $\left(\mathrm{m}^{3}\right)$. Los resultados mostraron una relación positiva entre media y considerable, entre estas dos variables $(r$-Pearson $=0,619$; $p$-valor $<0,001)$. En promedio, se observó un incremento del 5,0 \% en el IANC por cada 100000 m3 de AP. El modelo de regresión lineal obtenido fue el siguiente (ecuación (2)):

$$
\mathrm{IANC}=0,00005^{*} \mathrm{AP}-11,7
$$

Donde IANC representó el índice de agua no contabilizada (\%), y AP, el volumen de agua producida en el sistema de abastecimiento $\left(\mathrm{m}^{3}\right)$.

Por otro lado, los resultados sugirieron una relación positiva de débil a media $(r$-Pearson $=0,318$; $p$-valor $=0,002$ ) entre el AP y AF. Es decir, a medida que se incrementó la disponibilidad de agua en el sistema de abastecimiento probablemente se incrementó también el volumen de agua facturado. No obstante, esta relación fue menor en comparación con la relación entre el IANC y AP $(r$-Pearson $=0,619 ; p$-valor $<0,001)$. Las variables más significativas para estudiar la variación temporal del agua no contabilizada en el sistema fueron el IANC y AP (figura 1).

Al comparar el IANC con municipios de referencia, se observó que el sistema en estudio estuvo por debajo y por encima, en relación 
con otros sistemas de abastecimiento. En este estudio, el IANC promedio anual fue de $34,6 \%$, siendo inferior al reportado por los siguientes municipios de referencia: Soacha $(72,3 \%)$, Villavicencio $(67,0 \%)$, Sogamoso $(43,6 \%)$, Ibagué $(46,6 \%)$, Zipaquirá (48,2\%), Gachancipá $(36,7 \%)$ y Pitalito $(35,9 \%)$. Por el contrario, el IANC anual fue mayor en relación con los siguientes municipios: Tunja $(23,9 \%)$ y Mosquera $(21,3 \%)$. Los resultados mostraron que el IANC del sistema en estudio fue menor en comparación con siete de los nueve municipios de referencia seleccionados $(77,8 \%)$. En promedio en Colombia, el IANC fue de $45,3 \%$ entre los años 2007 y 2011 (Castillo, 2014) y 43,6\% para 2017 (DNP, 2018). De esta manera, los resultados sugirieron inicialmente un panorama favorable para el municipio en estudio en relación con el agua no contabilizada. No obstante,

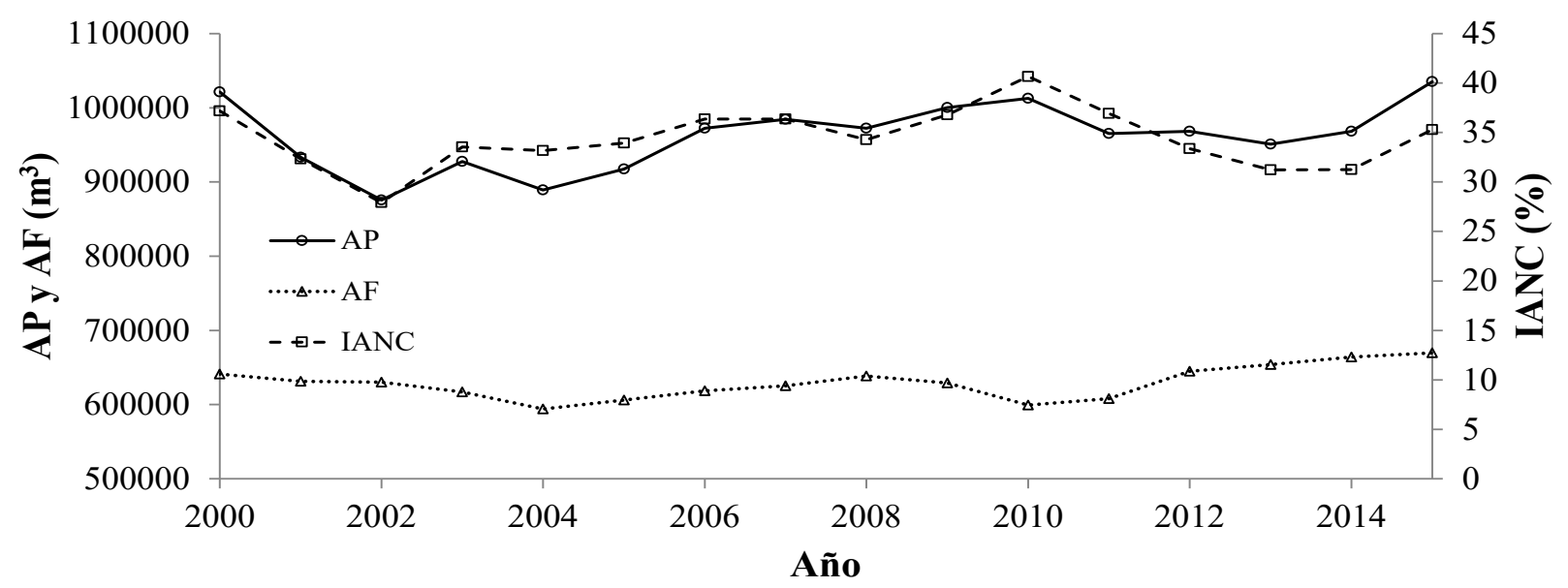

Figura 1. Variación promedio bimensual del IANC, AP y AF en el sistema de abastecimiento durante la totalidad del periodo de investigación

Fuente: elaboración propia.

el IANC máximo permisible por la normatividad colombiana fue de $30 \%$ (DNP, 2018). En países desarrollados el IANC tendió a 15\% y en países en vías de desarrollo tendió a 35\% (Kingdom, Liemberger y Marin, 2006. Al realizar un análisis comparativo anual en relación con el IANC por cada 100000 habitantes (IANC per cápita: IANCP) los resultados fueron diferentes. El IANCP promedio para el municipio de estudio fue de 30,7. Los resultados mostraron un mayor IANCP en los siguientes municipios de referencia: Gachancipá $(43,9)$, Pitalito $(46,3)$, Sogamoso $(44,4)$, y Zipaquirá $(43,4)$. Por otro lado, los siguientes municipios tuvieron un IANCP menor: Mosquera $(26,9)$, Soacha $(13,7)$, Villavicen- cio $(13,3)$, Ibagué $(8,65)$ y Tunja $(8,10)$. El IANCP en el sistema de estudio fue mayor en comparación con cinco de los nueve municipios de referencia (55,5\%). De esta manera, los resultados sugirieron un panorama menos favorable para el sistema de estudio en relación con el agua no contabilizada per cápita. Es decir, el acueducto probablemente experimentó dificultades técnicas y comerciales en la reducción de pérdidas debido a la magnitud de su IANCP; lo cual pudo ser una señal de la eficiencia con la que se prestó el servicio, así como de los costos que debieron asumir los usuarios del sistema. Tabesh et al. (2018) reportaron que las pérdidas económicas por agua no contabilizada fueron trasladadas di- 
rectamente a los usuarios facturados, elevando así los costos de suministro del agua. En relación con el índice de pérdidas por usuario facturado (IPUF), los resultados mostraron en promedio que, para el sistema de abastecimiento en estudio, este fue de $6,16 \mathrm{~m}^{3} /$ suscriptor*mes. En el orden nacional, se reportó en promedio un IPUF de 14,3 entre los años 2007-2011, con un rango de variación entre 3,20-33,5 (Castillo, 2014). Por ejemplo, los IPUF para Zipaquirá, Tunja y Mosquera para el año 2011 fueron de 2,20, 4,60 y 8,70, respectivamente. De esta manera, los resultados sugirieron un IPUF menor (56,9\%) en el sistema de estudio en relación con el promedio colombiano. En cuanto a Latinoamérica, por ejemplo, se han reportado IPUF para el año 2008 de 4,80, 9,30, 20,0 y 9,70 en las ciudades de Curiti- ba (Brasil), Santiago (Chile), Buenos Aires (Argentina) y Bogotá (Colombia), respectivamente (Kingdom, Liemberger y Marin, 2006). Por tanto, se evidenciaron IPUF superiores en la región latinoamericana, en comparación con el sistema en estudio. Por otro lado, se estudió la variación anual del consumo de agua en el sistema de abastecimiento con el objeto de evaluar su posible relación con el IANC. Los resultados indicaron que en 2000 y 2015 los usuarios facturados (UF) fueron 13801 y 26 576 , respectivamente. Los consumos de agua durante estos mismos años fueron 748 y 419l/ UF*día, respectivamente (figura 2). Es decir, las dotaciones de agua durante estos años fueron de 249,3 y 139,7l/habitante* día, respectivamente (promedio: 3 habitantes por UF).

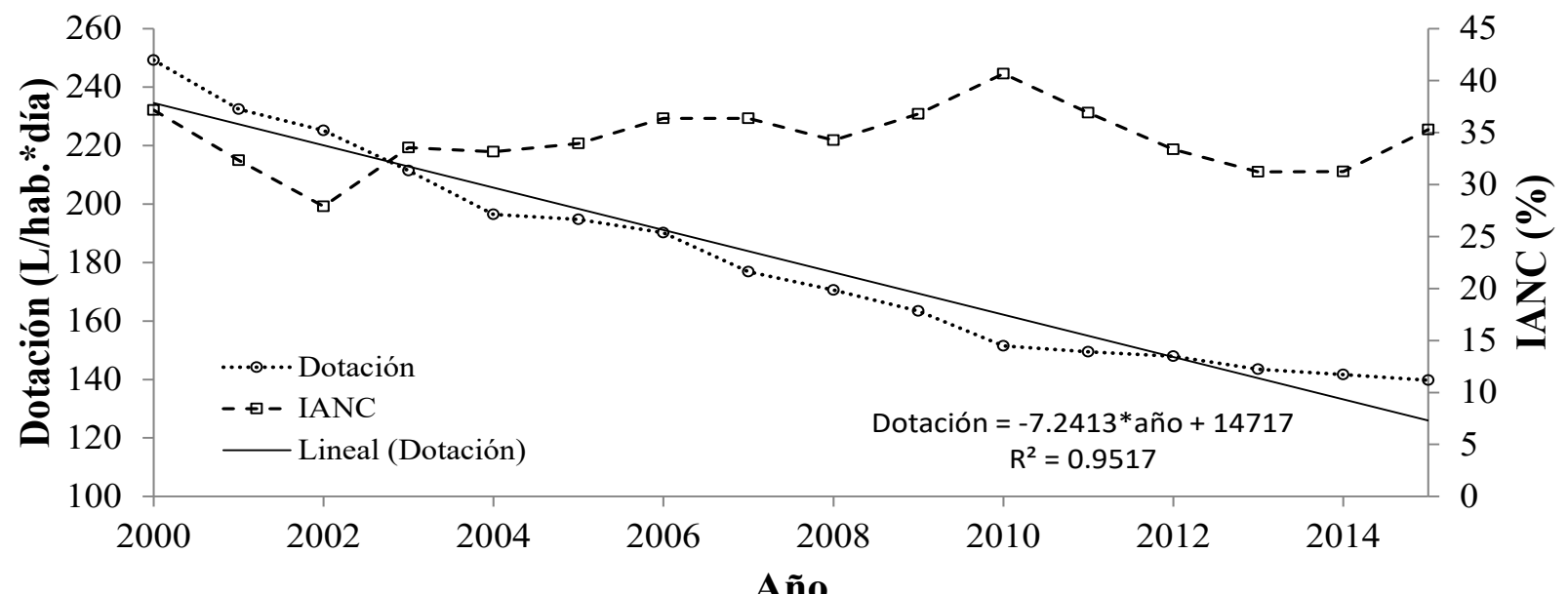

Figura 2. Variación anual promedio del IANC y la dotación en el sistema de abastecimiento de estudio. Modelo de regresión lineal para la dotación anual

Fuente: elaboración propia.

Esta última dotación coincidió con las recomendaciones de diseño dadas en la Resolución 2320 de 2009, por la cual se modificó parcialmente el RAS (140l/habitante*día) (MAVDT, 2009). De esta manera, los resultados sugirieron durante el periodo de estudio una disminución de la dotación del 43,9\%; en promedio, 7,24 I/habitante*año (2,93\%/ha- bitante*año). Sin embargo, el IANC no evidenció la misma tendencia decreciente (figura 2). Un análisis de correlación lineal de Pearson confirmó que no existió una relación significativa entre la dotación y el IANC ( $p$-valor $=0,497)$. Al parecer, el sistema de abastecimiento en estudio fue más efectivo durante las campañas de ahorro y uso eficiente del 
agua por parte de los UF, en comparación con las medidas adoptadas para disminuir las pérdidas técnicas y comerciales de agua (i.e., para disminuir el IANC).

\section{Influencia del régimen de precipitación}

Los resultados sugirieron que en el área de estudio las precipitaciones tuvieron un régimen bimodal, donde la época seca se registró entre enero y marzo, y agosto y septiembre $(\mathrm{P}<$ Q2, quintiles de Bolognesi); mientras que la época de lluvias se registró entre abril y julio, y octubre y diciembre ( $\mathrm{P} \geq \mathrm{Q} 3)$. En promedio, hubo una precipitación total anual de 789 $\mathrm{mm}$. Se observaron también periodos anuales de incremento y disminución de la precipitación posiblemente asociados con el fenómeno climático de Oscilación del Sur (EI Niño y La Niña). Al respecto, el Ideam (2014) reportó que durante los siguientes años se observaron eventos de La Niña con una intensidad fuerte en el área de estudio: 2007 y 2010. Estos eventos incrementaron la precipitación total anual. Durante el primer y segundo evento la precipitación anual se incrementó en 13,2\% y $32,6 \%$ con respecto de la precipitación anual promedio, respectivamente (figura 3 ). Según los periodos propuestos por el método de quintiles de Bolognesi (Bohn, Piccolo y Perillo, 2011), estos periodos correspondieron a muy lluviosos (Q3 $\leq \mathrm{P}<\mathrm{Q} 5)$. Durante estos periodos también se incrementó el IANC, en $4,05 \%$ y $18,5 \%$, respectivamente.

Por otro lado, la Unidad Nacional de Gestión del Riesgo de Colombia reportó que durante el periodo de estudio se observaron eventos de El Niño de intensidad moderada y fuerte en los años 2002 y 2014, respectivamente (UNGRD, 2016).

Estos eventos disminuyeron la precipitación total anual en el área de estudio. En promedio, durante el primer y segundo evento de
El Niño la precipitación disminuyó en 19,6\% y $10,5 \%$ con respecto de la precipitación promedio anual, respectivamente (figura 3 ). Estos periodos de tiempo correspondieron a muy secos $(\mathrm{P}<\mathrm{Q} 1)$, según el método de quintiles propuesto por Bolognesi (Bohn, Piccolo y Perillo, 2011). Durante estos periodos también se disminuyó el IANC, en 23,6 \% y $11,6 \%$, respectivamente.

A partir de lo anterior, mediante un análisis de Pearson se evidenció una relación positiva de considerable a muy fuerte ( $r$-Pearson $=0,812 ; p$-valor $<0,001)$ entre el IANC $(\%)$ y la altura total de precipitación $(\mathrm{mm})$. Los resultados sugirieron que a medida que se incrementó la precipitación también lo hizo el IANC (figura 3).Esta tendencia tal vez estuvo asociada con una mayor producción de agua en el sistema durante los periodos de incremento de la precipitación. Es decir, a medida que aumentó la disponibilidad de agua en el sistema también lo hizo el porcentaje de agua no contabilizada. En promedio, se observó un crecimiento del $2,28 \%$ en el IANC por cada incremento de $100 \mathrm{~mm}$ en la precipitación anual. El modelo de regresión lineal obtenido fue el siguiente (ecuación 3):

$$
\mathrm{IANC}=0,0228 * \mathrm{P}+16,4
$$

Donde IANC representó el índice de agua no contabilizada (\%), y P, la altura anual de precipitación $(\mathrm{mm})$.

\section{Análisis económico}

Uno de los análisis más importantes durante la evaluación del IANC fueron las pérdidas económicas que tuvo la empresa prestadora del servicio de agua en estudio. Al respecto, se utilizaron las estructuras tarifarias de 


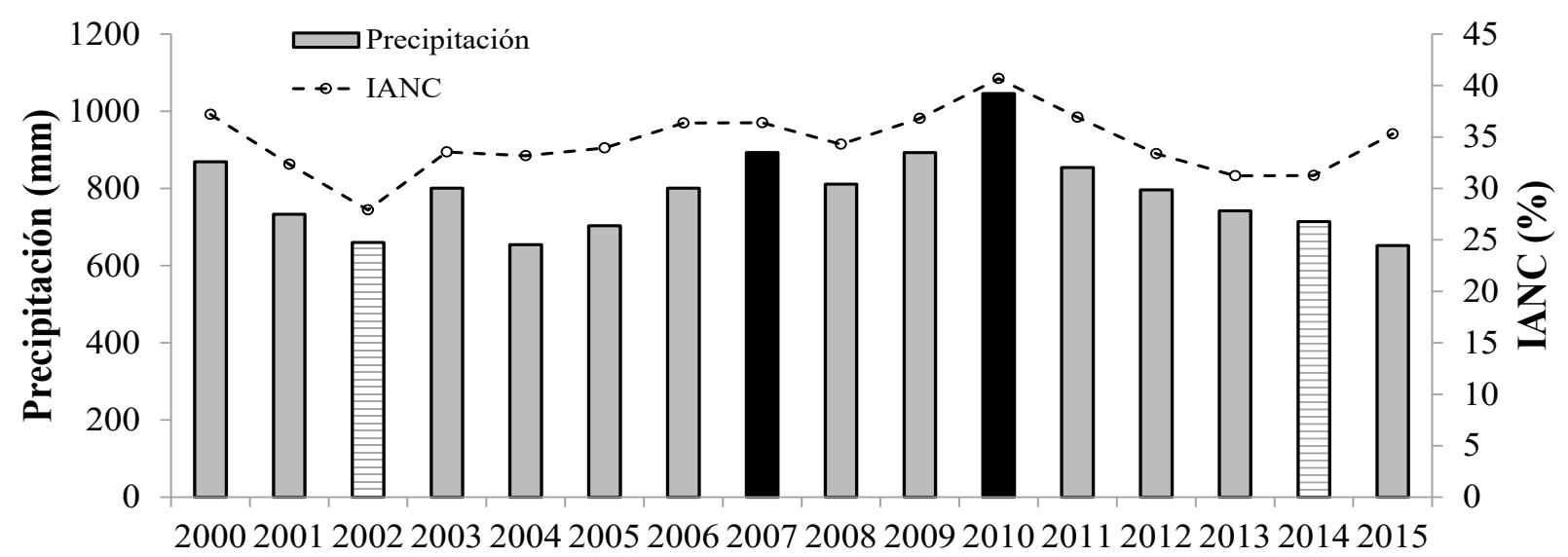

Año

Figura 3. Variación anual de la precipitación e IANC. Columnas negras: eventos fuertes de "La Niña". Columnas con líneas horizontales: eventos entre moderados y fuertes del "El Niño".

Fuente: elaboración propia.

agua potable y alcantarillado de seis países latinoamericanos de referencia (The World Bank, 2005): Perú, Chile, Colombia, Brasil, Argentina y México. El análisis económico se llevó a cabo con respecto de las tarifas de agua potable en la categoría doméstica o residencial normal. Esta categoría fue seleccionada debido a que fue la mayoritaria en la población de estudio (92,1\%).

El estrato socioeconómico seleccionado para este análisis fue el tipo dos (2), debido a fue el mayoritario en Colombia y en la población de estudio para un consumo promedio mensual de $20 \mathrm{~m}^{3}$ por UF (The World Bank, 2005).

La tabla 1, presenta los precios por $\mathrm{m}^{3}$ de AP en ciudades latinoamericanas y colombianas de referencia. Se muestran también las diferencias porcentuales con respecto al precio facturado por usuario en el sistema de abastecimiento en estudio. Según los resultados, en Argentina y Brasil se aplicaron los precios más bajos (USD 0,06 $\mathrm{m}^{3}$ ) y más altos (USD 0,91 $\mathrm{m}^{3}$ ) en Latinoamérica, res- pectivamente. El promedio de la región para los países de referencia fue de USD 0,44 $\mathrm{m}^{3}$, estando Colombia un 2,27\% por encima de este valor. En cuanto a la localidad de estudio, se registró un precio menor $(22,2 \%)$ con respecto al promedio latinoamericano de referencia. En el contexto colombiano, en promedio, hubo un precio por $\mathrm{m} 3$ menor (USD $\left.0,24 \mathrm{~m}^{3}\right)$ con respecto al sistema en estudio (USD 0,36 m3).

Es decir, en la localidad de estudio el precio por $\mathrm{m}^{3}$ de AF fue un 33,3\% mayor; el cual fue solo superado por el valor facturado en la ciudad de Bogotá (USD 0,38 m³).

Al relacionar el AP y AF durante todo el periodo de investigación (figura 1), según los resultados, en promedio se dejaron de contabilizar 29462400 m3, los cuales representaron una pérdida económica para el sistema de USD 10606464 para el año 2015. Según observación bimestral, el análisis económico prospectivo mediante modeIos ARIMA evidenció para el AP una estructura temporal: $p=3, d=0$ y $q=2$ (R2 $=0,413$; 
Tabla 1. Precio promedio de AP en países latinoamericanos y ciudades colombianas de referencia

\begin{tabular}{|c|c|c|}
\hline Localidad & Precio (USD/m³) & Diferencia $(\%)^{a}$ \\
\hline \multicolumn{3}{|c|}{ Latinoamérica } \\
\hline Brasil & 0,91 & $+152,8$ \\
\hline México & 0,57 & $+58,3$ \\
\hline Chile & 0,46 & $+27,8$ \\
\hline Colombia & 0,45 & $+25,0$ \\
\hline Perú & 0,22 & $-38,9$ \\
\hline Argentina & 0,06 & $-83,3$ \\
\hline Promedio latinoamericano & 0,44 & $+22,2$ \\
\hline \multicolumn{3}{|c|}{ Colombia } \\
\hline Ibagué & 0,14 & $-61,1$ \\
\hline Neiva & 0,14 & $-61,1$ \\
\hline Valledupar & 0,15 & $-58,3$ \\
\hline Pasto & 0,17 & $-52,8$ \\
\hline Popayán & 0,19 & $-47,2$ \\
\hline Bucaramanga & 0,19 & $-47,2$ \\
\hline Pereira & 0,21 & $-41,7$ \\
\hline Cúcuta & 0,25 & $-30,6$ \\
\hline Manizales & 0,25 & $-30,6$ \\
\hline Barranquilla & 0,26 & $-27,8$ \\
\hline Medellín & 0,27 & $-25,0$ \\
\hline Cartagena & 0,33 & $-8,30$ \\
\hline Cali & 0,35 & $-2,80$ \\
\hline Bogotá & 0,38 & $+5,60$ \\
\hline Promedio colombiano & 0,24 & $-33,3$ \\
\hline
\end{tabular}

a Diferencia porcentual en el precio por $\mathrm{m} 3$ con respecto de la localidad de estudio. Precio por m3 en la localidad de estudio para el año $2015=$ USD 0,36 m3

Fuente: elaboración propia, con información de The World Bank (2005).

Ljung-Box $\mathrm{Q}=0,059$; error absoluto promedio $=3,85 \%)$. Es decir, el AP en el sistema de abastecimiento en un instante de tiempo determinado estuvo influenciada por el AP durante los seis meses inmediatamente anteriores $(p=3)$. Los modelos ARIMA también indicaron una variabilidad corta en el AP. Es decir, en un instante determinado se sugirió una variación del AP durante los cuatro meses inmediatamente anteriores $(q=2)$. En relación con el AF, se registró, según observación bimestral, un modelo ARIMA: $p=0, d=1$ y $q=1$ 
$\left(R^{2}=0,371\right.$; Ljung-Box $Q=0,474$; error absoluto promedio $=4,14 \%$ ). Es decir, en un instante de tiempo determinado el AF no estuvo influenciada por el AF de los meses anteriores $(p=0)$. Igualmente, existió una variación del AF durante los dos meses inmediatamente anteriores $(q=1)$. De esta manera, el análisis mediante modelos ARIMA indicó un comportamiento temporal diferente para el AP y AF en el sistema. Finalmente, de acuerdo con los resultados ARIMA, en promedio, a corto (2016), mediano (2020) y largo plazo (2035) existirá una pérdida económica de USD 717 074, USD 3420279 y USD 13223 779, respectivamente (tabla 2).

Tabla 2. Precio promedio de AP en países latinoamericanos y ciudades colombianas de referencia

\begin{tabular}{|c|c|c|c|c|c|c|c|c|c|c|}
\hline Pronóstico & $\begin{array}{l}\text { Tiempo } \\
\text { (años) }\end{array}$ & $\begin{array}{c}\mathrm{Lla}-\mathrm{AP} \\
\left(\mathrm{m}^{3}\right)\end{array}$ & $\begin{array}{c}\text { LSb-APc } \\
\left(\mathbf{m}^{3}\right)\end{array}$ & $\begin{array}{c}\text { LI-AFd } \\
\left(\mathbf{m}^{3}\right)\end{array}$ & $\begin{array}{c}\text { LS-AF } \\
\left(\mathbf{m}^{3}\right)\end{array}$ & $\begin{array}{l}\text { LI-IAN- } \\
\text { Ce (\%) }\end{array}$ & $\begin{array}{c}\text { LS-IANC } \\
(\%)\end{array}$ & $\begin{array}{l}\text { LI-Costo } \\
\text { (USD) }\end{array}$ & LS-Costo (USD) & $\begin{array}{l}\text { Costo prome- } \\
\text { dio (USD) }\end{array}$ \\
\hline Corto & 1 & 881777 & 1109706 & 588204 & 739322 & 33,3 & 33,4 & 634118 & 800029 & 717074 \\
\hline Mediano & 5 & 862118 & 1098793 & 556191 & 771335 & 29,8 & 35,5 & 3304012 & 3536546 & 3420279 \\
\hline Largo & 20 & 849375 & 1090363 & 480580 & 846946 & 22,3 & 43,4 & 10515614 & 15931944 & 13223779 \\
\hline
\end{tabular}

${ }^{\mathrm{a}} \mathrm{LI}=$ límite inferior del pronóstico. ${ }^{\mathrm{b}} \mathrm{LS}=$ =límite superior del pronóstico; ${ }^{\mathrm{c}} \mathrm{AP}=$ agua producida; ${ }^{\mathrm{d}} \mathrm{AF}=$ agua facturada; ${ }^{\mathrm{e}}$

Fuente: elaboración propia.

\section{CONCLUSIONES}

Los resultados muestran que cuando crece la disponibilidad de agua al interior del sistema de abastecimiento (AP) probablemente también aumenta el IANC $(r=0,619)$. En promedio, se observa un incremento del $5,0 \%$ en el IANC por cada $100000 \mathrm{~m}^{3}$ de AP. También se sugiere al IANC y AP como las variables más adecuadas para estudiar la variación temporal del agua no contabilizada en el sistema. El régimen de precipitación tiene influencia sobre el IANC. Existe un aumento del 2,28\% en el IANC por cada incremento de $100 \mathrm{~mm}$ en la precipitación total anual $(r=0,812)$. Se evidenció un panorama favorable para el sistema cuando se utilizan como variables principales de estudio el IANC (34,6\%) e IPUF $\left(6,16 \mathrm{~m}^{3} /\right.$ suscriptor*mes). No obstante, es desfavorable cuando se utiliza como variable principal de estudio el IANCP (30,7\%/100.000 habitantes).

También, una evaluación integral de pérdidas de agua en sistemas de abastecimiento debe considerar índices adicionales al IANC. En este estudio se observa que no existe relación entre el IANC (\%) y la dotación (I/habitante*día). En efecto, según los resultados, la empresa operadora del sistema es tal vez más efectiva durante los programas de ahorro y uso eficiente del agua $(-43,9 \%)$ en comparación con los programas de reducción de pérdidas técnicas y comerciales $(34,6 \%)$. Estos dos programas deberían ser complementarios para gestionar integralmente el sistema de abastecimiento en estudio.

El análisis económico indica que el sistema en estudio posee un menor precio en el AP $(22,2 \%)$ con respecto a sistemas latinoamericanos de referencia. Sin embargo, presenta un mayor precio $(33,3 \%)$ en relación con el promedio colombiano. El análisis mediante modelos ARIMA señala una estructura temporal diferente para las series de tiempo de AP y AF. Es decir, los efectos del AP en un instante de tiempo determinado se transfieren durante seis meses (persistencia) en el sistema de abastecimiento; mientras que los efectos del AF no se transfieren en el tiempo. 
En promedio, el análisis económico prospectivo mediante modelos ARIMA muestra que a corto (2016), mediano (2020) y largo plazo (2035) existirá una pérdida de USD 717 074, USD 3420279 y USD 13223779 en el sistema, respectivamente.

Por último, este estudio permite ampliar el conocimiento en relación con el análisis de las pérdidas de agua en sistemas de abastecimiento urbano mediante el IANC, y será de utilidad para las empresas públicas y privadas prestadoras del servicio de acueducto. Lo anterior con el objeto de desarrollar programas de control de pérdidas, y de ahorro y uso eficiente del agua.

\section{FINANCIAMIENTO}

Esta investigación fue financiada por el Grupo de Investigación en Sistemas y Recursos Ambientales Sostenibles (Syras) de la Universidad Manuela Beltrán (Colombia), y fue apoyada académicamente por el Grupo de Investigación en Ingeniería Ambiental (GIIAUD) de la Universidad Distrital Francisco José de Caldas (Colombia).

\section{AGRADECIMIENTOS}

Los autores agradecen a la empresa Aguas de Facatativá E.S.P., por su apoyo logístico durante esta investigación.

\section{REFERENCIAS}

Agatón, A.L., Ruiz, J.C.C. y Sayago, U.F.C. (2016). Review of the status of art collection and utilization of rain water in urban and airports. Tecnura, 20(50), 141-153. DOI: https://doi.org/10.14483/22487638.11567

Bohn, V.Y., Piccolo, M.C. y Perillo, G.M.E. (2011). Análisis de los periodos secos y húmedos en el sudoeste de la provincia de Buenos Aires (Argentina). Revista de Climatología, 11(3), 31-43. Recuperado de http:// ri.conicet.gov.ar/handle/11336/21270

Box, G.E.P. y Jenkins, G. (1990). Time series analysis, forecasting and control. San Francisco, CA, EE. UU.: Holden-Day, Inc.

Boztaş, F., Özdemir, Ö., Durmuşçelebi, F.M. y Firat, M. (2019). Analyzing the effect of the unreported leakages in service connections of water distribution networks on non-revenue water. International Journal of Environmental Science and Technology, 16(8), 4393-4406. DOI: https://doi. org/10.1007/s13762-018-2085-0

Castillo, C.A. (2014). Marco tarifario para los servicios públicos de acueducto y alcantarillado (No. REGG-FOR07). Bogotá: Comisión de Regulación de Agua Potable y Saneamiento Básico Recuperado de https://tramitesccu.cra.gov.co/normatividad/ admon1202/files/3.\%20Documento\%20 de\%20trabajo\%20Perdidas.pdf

Cherchi, C., Badruzzaman, M., Gordon, M., Bunn, S. y Jacangelo, J.G. (2015). Investigation of cost and energy optimization of drinking water distribution systems. Environmental Science \& Technology, 49(22), 13724-13732. DOI: https://doi. org/10.1021/acs.est.5b03666

Departamento Nacional de Planeación (DNP) (2018). Estudio sectorial de los servicios públicos domiciliarios de acueducto y alcantarillado 2014-2017. Bogotá. Recuperado de https://www.superservicios.gov. co/sites/default/archivos/Publicaciones/ Publicaciones/2019/Ene/informe_sectorial-cuatrienio_2014-2017_.pdf

Díaz, Á.J.Á., Escobar, Y.C. y Serna, S.E.G. (2014). Análisis de la influencia de El Niño y La Niña en la oferta hídrica mensual de la cuenca del río Cali. Tecnura, 18(41), 
120-133. DOI: https://doi.org/10.14483/ udistrital.jour.tecnura.2014.3.a09

Dogo, E.M., Salami, A.F., Nwulu, N.I. y Aigbavboa, C.O. (2019). Blockchain and internet of things-based technologies for intelligent water management system. En F. Al-Turjman (ed.), Artificial Intelligence in IoT (pp. 129-150). Cham, Alemania: Springer. DOI: https://doi.org/10.1007/978-3030-04110-6_7

García, J.C. y Benavides, H. (2019). Adjustment value of water leakage index in infrastructure. DYNA, 86(208), 316-320. DOI: $\quad$ http://dx.doi.org/10.15446/dyna. v86n208.67230.

García, M., Vargas, C. y Granados, M. (2013). Estudio comparativo del índice de agua no contabilizada en Colombia para el periodo 1995-2011. Tecnogestión, 10(1), 26-35.

González-Gómez, F., García-Rubio, M.A. y Guardiola, J. (2011). Why is non-revenue water so high in so many cities? International Journal of Water Resources Development, 27(2), 345-360. DOI: https://doi.org/ 10.1080/07900627.2010.548317

Guerrero, V.M. (2003). Análisis estadístico de series de tiempo económicas. Barcelona: International Thomson Editores, S. A. de C. V.

Ho, C. I., Lin, M.D. y Lo, S.L. (2010). Use of a GIS-based hybrid artificial neural network to prioritize the order of pipe replacement in a water distribution network. Environmental Monitoring and Assessment, 166(14), 177-189. DOI: https://doi.org/10.1007/ s10661-009-0994-6

Instituto de Hidrología, Meteorología y Estudios Ambientales (Ideam) (2014). Actualización del componente meteorológico del modelo institucional del Ideam sobre el efecto climático de los fenómenos El Niño y La Niña en Colombia, como in- sumo para el Atlas Climatológico. Bogotá. Recuperado de http://www.ideam.gov. co/documents/21021/440517/Actualizacion+Modelo+Institucional+El+Ni\%C3\%B1o+-+La+Ni\%C3\%B1a.pdf/02f5e53b0349-41f1-87e0-5513286d1d1d

Kanakoudis, V. y Gonelas, K. (2016). Non-revenue water reduction through pressure management in Kozani's water distribution network: From theory to practice. Desalination and Water Treatment, 57(25), 11436-11446. DOI: https://doi.org/10.108 0/19443994.2015.1049967

Kingdom, B., Liemberger, R. y Marin, P. (2006). The challenge of reducing non-revenue water (NRW) in developing countries - how the private sector can help: A look at performance-based service contracting (No. 39405). Washington, D.C.: The World Bank. Recuperado de http://documents.worldbank. org/curated/en/385761468330326484/ The-challenge-of-reducing-non-revenuewater-NRW-in-developing-countries-howthe-private-sector-can-help-a-look-at-performance-based-service-contracting

Liemberger, R. y Wyatt, A. (2018). Quantifying the global non-revenue water problem. Water Supply, 19(3), 831-837. DOI: https://doi.org/10.2166/ws.2018.129

Ministerio de Ambiente, Vivienda y DesarroIlo Territorial (MAVDT) (2009). Resolución 2320. Bogotá. Recuperado de https:// www.funcionpublica.gov.co/eva/gestornormativo/norma.php?i=38487

Ministerio de Desarrollo Económico (MDE) (2000). Resolución 1096. Bogotá. Recuperado de https://www.alcaldiabogota.gov. co/sisjur/normas/Norma1.jsp?i=38541

Nazif, S., Karamouz, M., Tabesh, M. y Moridi, A. (2010). Pressure management model for urban water distribution networks. Water Re- 
sources Management, 24(3), 437-458. DOI: https://doi.org/10.1007/s11269-009-9454-x

Obras Sanitarias del Estado (OSE) (2019). Agua no contabilizada. Uruguay. Recuperado de http://www.ose.com.uy/agua/ agua-no-contabilizada

Ojeda, E. y Arias, R. (2000). Informe nacional sobre la gestión del agua en Colombia. Bogotá. Recuperado de https://www.cepal. org/drni/proyectos/samtac/inco00200.pdf

Pérez, S.E.B., Flores, S.M., González, O.N. y Mota, R.H. (2019). Implementación del método de escasez en la determinación de la huella hídrica en la zona costera de San Blas, México. Tecnura, 23(62). DOI: https://doi.org/10.14483/22487638.15796

Robayo, V., Castillo, D., Tangarife, I., Rojas, J., Puerto, C. y Villalba, N. (2017). Informe sectorial de acueducto y alcantarillado 2016. Bogotá: Superintendencia de Servicios Públicos Domiciliarios. Recuperado de https://www.superservicios.gov. $\mathrm{co} /$ ?q=publicaciones/acueducto-alcantarillado-y-aseo/informe-sectorial-acueducto-y-alcantarillado-2016

Salimi, A., Karami, H., Farzin, S., Hassanvand, M., Azad, A. y Kisi, O. (2018). Design of water supply system from rivers using artificial intelligence to model water hammer. ISH Journal of Hydraulic Engineering, $\mathrm{O}(0)$, 1-10. DOI: https://doi.org/10.1080/09715 010.2018 .1465366

Tabesh, M., Roozbahani, A., Roghani, B., Faghihi, N.R. y Heydarzadeh, R. (2018). Risk assessment of factors influencing non-revenue water using bayesian networks and fuzzy logic. Water Resources Manage- ment, 32(11), 3647-3670. DOI: https://doi. org/10.1007/s11269-018-2011-8

Tabesh, M., Yekta, A.H.A. y Burrows, R. (2009). An integrated model to evaluate losses in water distribution systems. Water Resources Management, 23(3), 477-492. DOI: https://doi.org/10.1007/s11269-0089284-2

The World Bank (2005). Las tarifas de agua potable y alcantarillado en America Latina (No. 32738). Washington, D.C. Recuperado de http://documentos.bancomundial. org/curated/es/398411468772501567/ Las-tarifas-de-agua-potable-y-alcantarillado-en-America-Latina

The World Bank (2013). América Latina: ¿Por qué las empresas de agua y saneamiento intentan ahorrar energía? Recuperado de https://www.bancomundial.org/es/news/ feature/2013/09/03/latin-america-water-loss-energy-efficiency

Unidad Nacional para la Gestión del Riesgo de Desastres (UNGRD) (2016). Fenómeno El Niño. Análisis Comparativo 19971998/2014-2016. Bogotá. Recuperado de https://repositorio.gestiondelriesgo.gov.co/ bitstream/handle/20.500.11762/20564/ Fenomeno_nino-2016.pdf? sequence $=3 \&$ isAllowed $=y$

Venkatesh, G. (2012). Cost-benefit analysis - leakage reduction by rehabilitating old water pipelines: Case study of Oslo (Norway). Urban Water Journal, 9(4), 277-286. DOI: https://doi.org/10.1080/1573062X.2012.660960

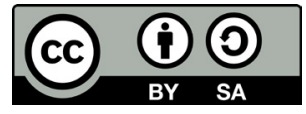

